\title{
Pathological Characteristics of Nasopharyngeal Carcinoma in Hue Central Hospital
}

Pham Nguyen Cuong, Nguyen Thanh Xuan, Pham Nguyen Tuong, Tran Thi Ngoc Phuong and Nguyen Thi Hoai An

Hue Central Hospital ,The Pathology Department of Hue Central Hospital, Hue city, Zip code: 530000, Vietnam.

\begin{abstract}
Materials and Methods: This was a retrospective review of all patients seen at Pathology Department, Hue Central Hospital with the diagnosis of nasopharyngeal carcinoma between January 1, 2017 and June 30, 2018. The patient's clinical data and histopathological findings are presented. The histopathological diagnosis was in accordance with the World Health Organization (WHO) 1991 classification table. Results: A total of 73 patients with histologically confirmed nasopharyngeal carcinoma were recorded at Hue Central Hospital. There were 53 males and 20 females. Histologically, undifferentiated carcinoma was the commonest (71.2\%) followed by well-differentiated keratinizing squamous cell carcinoma (20.6\%) and other carcinoma (8.2\%). Immunohistochemistry with CKAE $1 / 3$ was positive to $32 / 32$ of well-differentiated keratinizing squamous cell carcinoma, and negative to $30 / 32$ of undifferentiated carcinoma.
\end{abstract}

Key words: Nasopharyngeal Carcinoma, Pathological Characteristics.

\section{Background}

Nasopharyngeal cancer is a type of cancer that comes from the epithelial mucosa in the nasopharynx area. Nasopharyngeal cancer is the most common cancer among head and neck cancer and is one of the ten most common cancers in Vietnam [1]. In Hue Central Hospital, the rate of nasopharyngeal cancer is increasing and the cancer age of this type is getting younger. The specific study of pathological characteristics and immunohistological characteristics of this type of cancer in Hue Central Hospital has not been much. We carried out the topic: "Pathological Characteristics of Nasopharyngeal Carcinoma in Hue

Corresponding author: Pham Nguyen Cuong, Ph.D., M.D., academic title if any, research fields: Research field: Lung cancer, Nasopharyngeal cancer, Immunohisstochemistry markers in Viet nam.
Central Hospital" to learn more about this problem, for diagnosis and treatment better.

\section{Patients and Method}

The study of 73 patients recorded at Pathology Department, Hue Central Hospital with the diagnosis of nasopharyngeal carcinoma, picked up between January 1, 2017 and June 30, 2018. The patient's clinical data, histopathological and immunohistochemistry results are presented. The histopathological diagnosis was in accordance with the World Health Organization (WHO) 1991 classification.

Data were processed using SPSS 16.0 for Microsoft Excel 2003.

\section{Results}

\subsection{Patients Characteristics}




\subsubsection{Gender}

Table 1 Gender.

\begin{tabular}{lll}
\hline Gender & Number & $\%$ \\
\hline Male & 50 & $68.5 \%$ \\
Female & 23 & $31.5 \%$ \\
Total & 73 & $100 \%$ \\
\hline
\end{tabular}

Men are common for about 2 times than women.

\subsubsection{Age Groups}

Table 2 Age groups.

\begin{tabular}{lll}
\hline Age groups & Number & $\%$ \\
\hline$<30$ & 8 & $11 \%$ \\
$30-55$ & 40 & $54.8 \%$ \\
$\geq 55$ & 25 & $34.2 \%$ \\
Total & 73 & $100 \%$ \\
\hline
\end{tabular}

The age group from 30 to 55 years old accounts for the highest percentage. Youngest: 27 years old, the oldest: 89 years old.

\subsection{The Histopathological Diagnosis was according to the WHO 1991 Classification}

Table 3 The Histopathological Diagnosis was according to the WHO 1991 Classification.

\begin{tabular}{lll}
\hline WHO 1991 & Number & $\%$ \\
\hline Undifferentiated carcinoma & 52 & $71.2 \%$ \\
Well differentiated carcinoma & 15 & $20.6 \%$ \\
Other carcinoma & 6 & $8.2 \%$ \\
Total & 73 & $100 \%$ \\
\hline
\end{tabular}

\subsection{Immunohistological Characteristics}

Table 4 Immunohistological Characteristics.

\begin{tabular}{lll}
\hline Histopathology type & CKAE1/3 positive & $\%$ \\
\hline Undifferentiated carcinoma & 2 & $6.25 \%$ \\
Well differentiated carcinoma & 32 & $100 \%$ \\
Other carcinoma & 0 & $0 \%$ \\
\hline
\end{tabular}

\section{Discussion}

Our research found that in nasopharyngeal carcinoma, men has a higher rate than women. This rate is consistent with other studies in the country as well as abroad, the rate of nasopharyngeal carcinoma in men is higher than that of women $[1,4,5]$.

In this study, the youngest was 28 years, the highest was 89 years. The age group from 30 to under 55 years old has the highest proportion (54.8\%). This result is also similar to the research of Nghiem Duc Thuan [5].
According to Nguyen Thi Bich Ha, 1993, the highest age between 40 and 60 years old is 58.33\% [6]. According to Shan R., Bake's average age of nasopharyngeal carcinoma is 48.4 years [7]. According to Dickson R. I., patients over 40 years old accounted for $69 \%$, under 10 years old is only $1 \%$ [8].

The diagnosis according to the WHO 1991, the nasopharyngeal carcinoma, the undifferentiated nasopharyngeal carcinoma accounts for the highest rate $(71.2 \%)$, the well-differentiated carcinoma accounts for $20.6 \%$, the another type of cancer is $8.2 \%$. Nghiem 
Duc Thuan's research showed that the undifferentiated nasopharyngeal carcinoma accounted for the highest rate of $88.89 \%$, also applied according to WHO 1991's histological classification table, showed that the rate of undifferentiated cancers in cancer is highest.

About immunohistochemistry: we use the CKAE1/3 marker in the nasopharyngeal carcinoma to study the reveal of immunohistochemistry markers in each type of histology of this cancer. Accordingly, the well-differentiated nasopharyngeal carcinoma is positive for CKAE1/3 marker is $100 \%$, while the nasopharyngeal carcinoma and other types of cancer are low. This is the same to the literature because the CKAE1/3 marker is a marker of epithelial cell specificity. The negative of un differentiated and another type of cancer for CKAE1/3 marker in this case is explainable and therefore, staining immunohistochemistry with CKAE1/3 marker in this case is for the differential diagnosis from the well-differentiated squamous carcinoma and the remaining types of nasopharyngeal carcinoma, because some cases are very difficult to diagnose with traditional Hematoxylin-Eosin (HE) staining.

\section{Conclusion}

The proportion of men suffering from nasopharyngeal carcinoma is nearly two times higher than that of women.
The type of undifferentiated carcinoma is common in nasopharyngeal carcinoma.

$100 \%$ case of nasopharyngeal carcinoma is positive CKAE1/3 marker in well-differentiated squamous carcinoma, and negative almost in undifferentiated carcinoma. The use of immunohistochemistry is effective when it is necessary to diagnose the origin of the type of cancer cell in metastatic lymph nodes, or to confirm the type of cancer of the nasopharyngeal carcinoma in the case of difficult diagnosis with the HE staining.

\section{Requesting}

Most cancerous tissue pieces are from a biopsy. It is recommended that the specimen should get the right and enough area of injury, avoid taking the necrosis area and taking the edge of the lesion. The clinical doctors, of the Otolaryngology Department and the Oncology Department should have a consult for determinating the location and general characteristics of the lesion for diagnosis and prognosis.

Nasopharyngeal carcinoma is a very dangerous disease and difficult to detect, so the only way to detect signs of cancer in general and nasopharyngeal carcinoma in particular is screeening for lesion. Every six months or when there are signs of sore throat, the patient should be checked by the experts to have the right diagnosis and treatment.

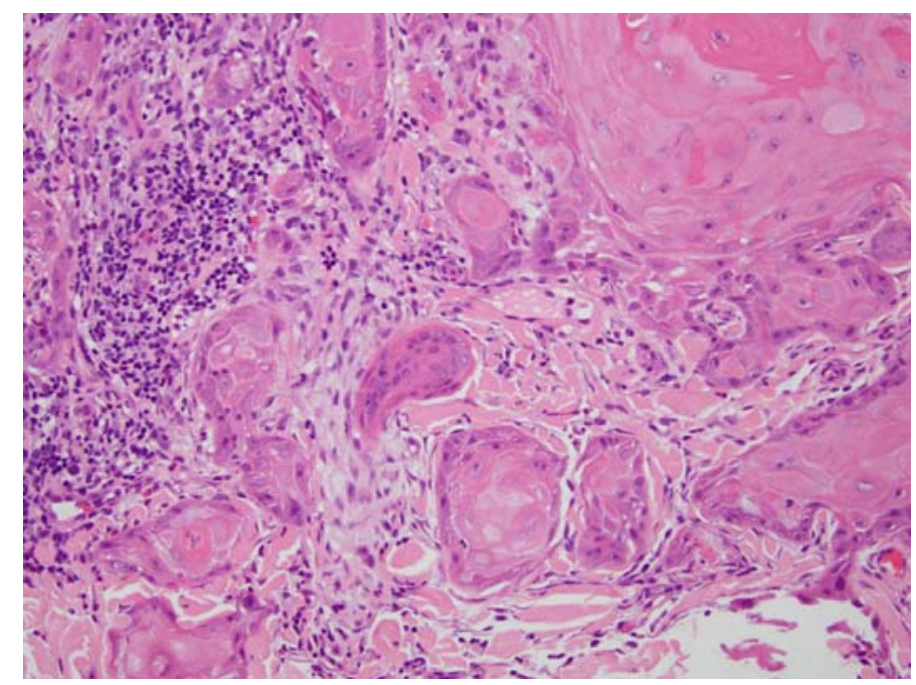

Fig. 1 Well-differentiated carcinoma. H.E $\times 400$. 


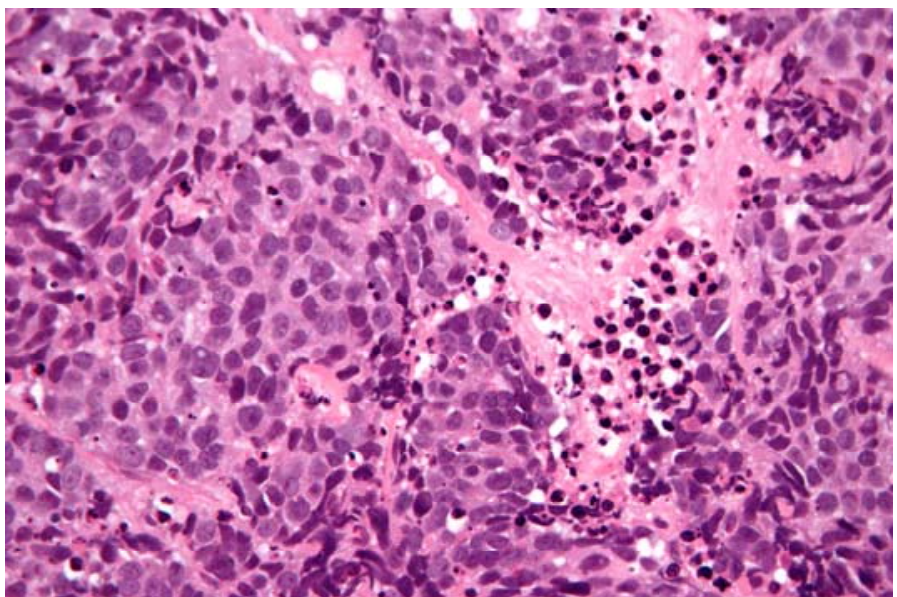

Fig. 2 Undifferentiated carcinoma, H.E $\times 400$.

\section{Reference}

[1] Nguyen, C. H. 2001. "Tobacco and Cancer.” Ho Chi Minh City's Medicine, a Sub-Topic on Oncology 5 (4): 1-5.

[2] Thinh, D. H. Q. 2005. "Results of Treatment of Cervical Cancer by Combining New Supportive Chemotherapy and External Radiation Therapy." Specialist thesis of Level II-Oncology, Ho Chi Minh City University of Medicine and Pharmacy.

[3] Nguyen, S. T. 1992. The Upper Gastrointestinal Tract Tumor, edited by Nguyen Sao Trung, Nguyen Chan Hung, Basic Oncology Pathology, Center for Training and Retraining Medical Staff, HCMC, 29-44.
[4] Young LS $^{1}$, Dawson CW, Clark D, Rupani H, Busson P, Tursz T, Johnson A, Rickinson AB, 2003 "Study of Epstein Bar Virut Gene Activity in Nasopharyngeal Tumor Biopsy". Practical Medicine Journal, Oncology topic, 7 (4): 29.

[5] Nguyen, T. B. H. 1993. "The Situation of Relapse of Throat Cancer after Radiotherapy Treatment at 108" Hospital of Medicine, Vietnam Medicine, 9/1994, 184, p. 27-36.

[6] Baker, S. R. 2006. "Prognostic Factors of Nasopharyngeal Malignancy." Version of Record Online, June 28, 2006.

[7] Dickson, R. I. 1986. "Cancer of the Nasopharynx in British Columbia.” Am J Clin Oncol. 9 (4): 281-91. 